\section{P3.080 PREVALENCE OF C. TRACHOMATIS, N. GONORRHEAE, T. VAGINALIS, C. ALBICANS, SYPHILIS, BACTERIAL VAGINOSIS \& HIV IN A POPULATION OF WOMEN WITH SYMPTOMS OF LOWER GENITAL INFECTIONS IN BOGOTÁ, COLOMBIA 2010}

doi:10.1136/sextrans-2013-051184.0540

${ }^{1} E$ Angel Muller, ${ }^{2} \mathrm{~A}$ Rodriguez, ${ }^{3} \mathrm{~L}$ Nuñez-Forero, ${ }^{4} \mathrm{~L}$ Moyano, ${ }^{3} \mathrm{P}$ González, ${ }^{3} \mathrm{E}$ Osorio, ${ }^{5} \mathrm{~L}$ Diaz, ${ }^{4} \mathrm{~N}$ Rodriguez, ${ }^{6} \mathrm{~A}$ Ruiz Parra, ${ }^{6} \mathrm{H}$ Gaitan Duarte. ${ }^{1}$ Department of Obstetrics and Gynecology, National University of Colombia., Bogota, Colombia; ${ }^{2}$ Clinical Research Institute, National University of Colombia, Bogota, Colombia; ${ }^{3}$ Health Secretariat, Bogota, Colombia; ${ }^{4}$ Department Statistics, Universidad Nacional de Colombia, Bogota, Colombia; ${ }^{5}$ Department of Obstetrics and Gynecology, Universidad Nacional de Colombia, Bogota, Colombia; ${ }^{6}$ Clinical Research Institute, Universidad Nacional de Colombia, Bogota, Colombia

Objetives To determine the prevalence and aetiology of lower genital tract infections (LGTIs) in symptomatic women of reproductive age and describe the risk factors.

Methods Cross sectional study. Symptomatic women who consulted at three ambulatory care centres in Bogotá, Colombia. Exclusions: pregnancy, hysterectomy, antibiotics in the previous 7 days. Samples were obtained for etiologic diagnosis using gold standard tests for: bacterial vaginosis (VB) by Nugent's criteria; blood agar culture for Candida and a wet smear and In pouch ${ }^{\mathrm{TM}}$ culture for $\mathrm{T}$. vaginalis. PCR for C. trachomatis (CT) and N. gonorroheae (NG), and serologic tests for syphilis (RPR, TPHA) and HIV.

Results 1385 women were recruited from February to December $2010.115(8.3 \%)$ of them were sexual workers. A LGTI was confirmed in $731(52.7 \%) ; 560(40.4 \%)$ had an endogenous infection and 170 $(12.3 \%)$ a sexually transmitted infection (STI). The most frequent aetiology was VB in 549 (39.6\%), followed by candidiasis in 153 (11\%). CT was detected in 134 (9.7\%), NG in 19 (1.4\%), Trichomonas by wet smear in $11(0.8 \%)$ and by culture in 8/634 (1.2\%), syphilis in $12(0.8 \%)$ and HIV in 1 case. The risk factors of STI are: sex workers (OR: 2.0, CI $95 \% 1.2-3.3)$, younger age ( $28 \pm 7.8$ vs. $32 \pm 8.9$ (mean \pm SD), no health insurance (23.5 vs. $15.4 \%$ ) and alcohol users (OR: 2.6 (95\% CI: $1.4-4.5)$ ) Conclusions: $52.7 \%$ of women who consult for symptoms of LGTIs an aetiology can be identified, being BV the most common and Chlamydia the most frequent STI. Almost the same number of women (47.3\%) has no specific aetiology identified, even with the use of gold standard diagnostic technology. This information should be used by policy makers and clinicians for prioritisation of prevention and diagnosis of LGTIs and use of syndromic management

\section{P3.081 TIME TO SEXUALLY TRANSMITTED INFECTIONS AFTER NEGATIVE STI TESTING AMONG YOUNG ADULTS}

doi:10.1136/sextrans-2013-051184.0541

${ }^{1}$ T Anderson, ${ }^{2} \mathrm{D}$ Hensel, ${ }^{2} \mathrm{~F} \mathrm{He},{ }^{2} \mathrm{~J}$ Harezlak, ${ }^{1} \mathrm{~J}$ Fortenberry. ${ }^{1}$ Indiana University School of Medicine, Indianapolis, IN, United States; ${ }^{2}$ Indiana University Fairbanks School of Public Health, Indianapolis, IN, United States

Background Recommendations for the frequency of STI screening in high-risk populations are limited by lack of data about when infections occur following a negative screening test.

Methods Participants (18-29 years of age; women [N=192] and men $[N=156]$ ) in a 12 -week study of incident STI were recruited from a county STI clinic. Self-collected vaginal samples (women) and urine samples (men) were obtained weekly at participant's home. Nucleic acid amplification tests (NAAT) were used for diagnosis of $C$. trachomatis, $N$. gonorrhoeae, and $T$. vaginalis infections. Infections diagnosed at enrollment were treated immediately. Based on cumulative diary reports of partner-specific sexual behaviours, an exposure variable was created to indicate vaginal exposure with only one partner, or with more than one partner. Time to infection was modelled using Kaplan-Meier curves; group differences were assessed via logrank test. Logistic regression was used to assess factors associated with infection status at the study completion.

Results 146/192 (76\%) women and 126/156 (81\%) men were uninfected at enrollment. At 4 weeks post-enrollment, $16 \%$ of women and $8 \%$ of men had at least one STI. At 8 weeks post-enrollment, about $23 \%$ of women and $10 \%$ of men were infected. Overall, $54 / 192$ (28\%) women and 19/156 (12\%) men had at least one STI during the 12-week followup. Survival curves differed significantly by gender $(p=0.0003)$. In a multivariable logistic model, women had significantly higher odds of STI (odds $=3.3, p=0.004$ ), controlling for infection status at enrollment and monogamy status. Gender remained significant in models including number of partners during the follow-up period.

Conclusion In high STI-risk populations, rescreening of initially negative persons identifies high subsequent infection rates, especially among women. Early rescreening (e.g. within 3 months) of any tested person would significantly increase identification of reinfections among initially positive and treated as well as new infections among those previously testing negative.

\section{P3.082 INCIDENCE OF CONGENITAL SYPHILIS IN PARAGUAY}

doi:10.1136/sextrans-2013-051184.0542

C Aranda, G Aguilar, Z Suarez, S Munoz, C Fusillo, A Kawabata. National Program of Control of HIVAidsSTI, Asuncion, Paraguay

Background The objective was to determine the incidence of congenital syphilis cases in the public health sector of Paraguay in 2010. Methods observational, descriptive, retrospective study of newborns of the services of the country's public health sector excluding the Chaco region. The sample was obtained through two stage cluster sampling. The unit of analysis are the newborns.

Results Of 6739 newborn registries examined, 69 met the criteria for definition of congenital syphilis case. The incidence of congenital syphilis was 10.23 per 1000 live births. $73.9 \%$ of the mothers of the children with congenital syphilis had attended the prenatal check-ups. In $37.7 \%$ of pregnant women syphilis was diagnosed less than a month before childbirth followed by $26.1 \%$ that were diagnosed during labour and did not receive treatment. Two of the 69 newborns died.

Conclusion Congenital syphilis continues to be an important public health problem in Paraguay. The majority of the women with children with congenital syphilis attended prenatal check-ups, indicating missed opportunities for the health services. The high incidence of the congenital syphilis is an indicator of the quality of the health services and this highlights the need to consider syphilis among the priority problems in Paraguay, and to intensify actions for its control.

\section{P3.083 IS THE SYPHILIS INCIDENCE DECREASING IN GEORGIA?}

doi:10.1136/sextrans-2013-051184.0543

${ }^{1,2} \mathrm{G}$ Galdava, ${ }^{\prime} \mathbf{O}$ Kulividze, ${ }^{3} \mathrm{M}$ Unemo, ${ }^{4} \mathrm{M}$ Domeika, Eastern European Network for SexualReproductive Health. ' ${ }^{S} / R$ National Center of Dermatology and Venereology, Tbilisi, Georgia; ${ }^{2}$ Faculty of Medicine, Tbilisi State University, Tbilisi, Georgia; ${ }^{3}$ WHO Collaborating Centre for Gonorrhoea and other STIS, Örebro, Sweden; ${ }^{4}$ Department of Control and Prevention of Communicable Diseases Uppsala County Council, Uppsala, Sweden

Background The epidemiology of syphilis in Georgia has traditionally been determined by socio-economic factors such as low solvency of Georgian population, urban migration, level of financing of STI prevention programmes etc. This study reports on the epidemiology of syphilis in Georgia over the period of 2000-2011.

Methods Analysis of 12 years data on syphilis incidence provided by the National Center for Diseases Control and Public Health, Tbilisi, Georgia. 